\title{
EPR linewidth of local magnetic moments diluted in Ce intermediate-valence compounds
}

\author{
Pablo A. Venegas \\ Departamento de Física, Faculdade de Ciências, UNESP, Campus Bauru, R. Engenheiro Luis Coube s/n, Bauru, São Paulo, Brazil \\ Gaston E. Barberis \\ Instituto de Física, Universidade Estadual de Campinas (UNICAMP), 13081 Campinas, São Paulo, Brazil
}

(Received 23 December 1991)

\begin{abstract}
Anomalous thermal behavior on the EPR linewidths has been observed for Gd impurities diluted in $\mathrm{Ce}_{x} A_{1-x} B_{n}(A=\mathrm{La}, \mathrm{Y}, B=\mathrm{Ir}, \mathrm{Os}, \mathrm{Rh}, \mathrm{Pd})$ intermediate-valence compounds. In this work we show that the exchange interaction between the local magnetic moments and the intermediate-valence host ions has an important contribution to the relaxation rates of the local moments. We calculated the relaxation, using the Redfield formalism and the ideas contained in the interconfigurational fluctuation model of Hirst. We show that the exchange interaction contribution has an exponential dependence on the excitation energy of the intermediate-valence ions.
\end{abstract}

\section{INTRODUCTION}

Ce intermetallic compounds have been extensively studied in the literature and many of them show anomalies typical of intermediate-valence (IV) alloys. ${ }^{1}$ By doping $\mathrm{Ce}$ compounds with $\mathrm{Gd}^{3+}$ ions, their ESR spectra allow us to study locally the influence of the intermediate-valence $\mathrm{Ce}$ ions. In metals, the local moments ESR linewidth, $\Delta H$, is expected to increase linearly with the temperature (Korringa ${ }^{2}$ ). In contrast, in $\mathrm{Ce}_{x} A_{1-x} \mathrm{Pd}_{3}(A=\mathrm{Ag}, \mathrm{Y})$ and $\mathrm{Ce}_{x} \mathrm{La}_{1-x} \mathrm{Os}_{2}$ the Gd ESR spectra show a nonlinear increase of $\Delta H$ in the temperature range $4.2 \leq T \leq 300 \mathrm{~K}^{3}$ At low temperatures the slope $d(\Delta H) / d T$ is smaller than in the isostructural nonIV compounds $M \mathrm{Pd}_{3}(M=\mathrm{Sc}, \mathrm{Y}, \mathrm{La}),{ }^{4}$ whereas at high temperatures the resonance line is strongly broadened and the slope asymptotically approaches the value measured in $\mathrm{LaPd}_{3}$. The temperature dependence of $\Delta H$ becomes nonlinear and cannot be explained only by invoking the usual Korringa mechanism.

EPR studies in metallic hosts, which show interconfigurational fluctuations, ${ }^{5}$ suggest that the indirect exchange interaction between the local magnetic impurities and the Ce $4 f$ electrons have an appreciable contribution to the linewidth of the magnetic impurities. The $\mathrm{Ce}$ interconfigurational fluctuations are transferred via the Ruderman-Kittel-Kasuya-Yoshida (RKKY) interaction to the $\mathrm{Gd}$ site. These fluctuations generate an effective alternating magnetic field, which relaxes the $\mathrm{Gd}$ spins. A similar mechanism was also used for the interpretation of the NMR relaxation rates of noble metals with Kondo impurities, ${ }^{6-9}$ for perturbed angular correlation measurements of ${ }^{140} \mathrm{Ce}$ in $\left(\mathrm{La}_{1-x} \mathrm{Y}_{x}\right) \mathrm{Al}_{2}$ alloys, ${ }^{10}$ and for Gd ESR on concentrated Van Vleck paramagnets. ${ }^{11}$ We use this mechanism to explain the anomalous behavior of the linewidth of $\mathrm{Gd}$ diluted in the $\mathrm{Ce}$ intermediate valence compounds.

The Gd linewidth can be calculated with use of the ideas contained in the "interconfigurational fluctuation" model of Hirst. ${ }^{12-14}$ This model has been used to explain various physical measurements and particularly magnetic susceptibility and Mössbauer studies in intermediatevalence compounds. In their approach the expectation value of the measured physical quantity (isomer shift, magnetic moment, etc.) is calculated by "averaging" over all possible $4 f^{n}$ configurations. The occupation probability of the $n$ configuration is usually expressed as $P_{n}(T) \propto \exp \left(-E_{n} / T_{n}\right)$ with $T_{n}=T+\Delta_{n} \cdot 1,14,15$ Here $E_{n}$ and $\Delta_{n}$ are the energy and width of the $4 f^{n}$ configuration. The quantity measured thus depends exponentially on the excitation energy, $E_{\text {ex }}$, defined as the energy required to delocalize a single electron from the $4 f^{n}$ configuration (i.e., $E_{\mathrm{ex}}=E_{n}-E_{n-1}$ ). Although this theoretical approach is phenomenological, it successfully explains most of the Europium Mössbauer spectra and susceptibility studies in mixed-valence compounds. ${ }^{16,17}$

In this work we use this approach and the Redfield formalism $^{18}$ to calculate the contribution of an excited configuration of the host $\mathrm{Ce}$ ions to the linewidth of the impurity spectra. We fit the experimental data and compare with other theoretical approaches. ${ }^{3,19}$

\section{THE MODEL}

The spin Hamiltonian describing the $\mathrm{Ce}$ ions in the $n$ configuration can be written

$$
H=H_{\mathrm{cf}}+H_{z},
$$

where the crystal-field Hamiltonian for $\mathrm{Ce}$ is

$$
H_{\mathrm{cf}}=\sum_{l}\left[B_{4} O_{4}\left(\mathrm{~J}^{(l)}\right)+B_{6} O_{6}\left(\mathrm{~J}^{(l)}\right)\right]
$$

with $\mathrm{O}_{4}\left(\mathbf{J}^{(l)}\right)$ and $\mathrm{O}_{6}\left(\mathbf{J}^{(l)}\right)$ the cubic combinations of Stevens' equivalent operators of the fourth and sixth degree, respectively, $B_{4}$ and $B_{6}$ are the crystalline-field parameters of the fourth and sixth degree, respectively, and $\mathbf{J}^{(l)}$ is the total angular momentum of the $l$ th $\mathrm{Ce}$ ion. The Zeeman Hamiltonian for $\mathrm{Ce}$ is

$$
H_{z}=g_{J} \mu_{B} \sum_{l} \mathbf{H} \cdot \mathbf{J}^{(l)},
$$


where $\mu_{B}$ is the Bohr magneton, $g_{J}$ is the Lande factor, and $\mathbf{H}$ is the external magnetic field.

The Gd Hamiltonian can be written as

$$
H^{\prime}=H_{\mathrm{cf}}^{\prime}+H_{z}^{\prime}+H_{\mathrm{ex}} \text {. }
$$

Here $H_{\mathrm{cf}}^{\prime}$ and $H_{z}^{\prime}$ are defined in a similar form as $H_{\mathrm{cf}}$ and $H_{z}$, with the obvious substitution of $\mathbf{J}^{(l)}$ by the spin of the Gd, S. The Gd impurity interacts with the neighboring host $\mathrm{Ce}$ ions via exchange interaction:

$$
H_{\mathrm{ex}}=-\left(g_{J}-1\right) \partial \sum_{l=1}^{z_{0}} \mathbf{S} \cdot \mathbf{J}^{(l)},
$$

where $\alpha$ is the exchange intergal between the two ions and $z_{0}$ is the number of Ce neighbor ions of each impurity. For simplicity, however, we have considered only the first Ce neighbors. We calculate the relaxation rate of the impurity if it does not create a perturbation of the $\mathrm{Ce}$ host Hamiltonian.

\section{THE LINEWIDTH}

In our metallic host the impurity ESR linewidth calculations must consider the energy transfer between the impurity spin $S$, the spin of the host rare-earth ions $(\mathrm{Ce})$, the spins of the conduction electrons as well as the lattice, in the presence of static external magnetic field, and a small alterating field. In the present work we shall assume that the system is in the unbottlenecked regime, i.e., the conduction electrons and the host magnetic ions are in equilibrium with the lattice. In addition we shall assume that the transverse susceptibility associated with the impurity at the impurity resonance frequency, $\omega_{0}$, is much larger than those of the other spin systems at the same frequency. In this limit one can consider only the transverse magnetization, $M_{x}$, of the impurities and neglect the interaction of the $r f$ field with the magnetic host ions and the conduction electrons. In other words, the magnetic host ions and the conduction electrons are "passive dissipative systems." On the other hand, the static magnetic field splits the Gd resonance in $2 S=7$ fine-structure lines, which are well resolved at low temperatures. ${ }^{20}$ In the present case, for simplicity, we assume that the fine structure is collapsed and suppose the Gd with an effective spin $S=\frac{1}{2}$. With these assumptions the impurity linewidth can be expressed as

$$
\Delta H=a+b T+\Delta_{I F},
$$

where $a$ is the residual linewidth, $b$ is the usual Korringa contribution originating from the impurity-conductionelectron exchange interaction, and $\Delta_{I F}$ is the impurity linewidth due to the exchange interaction with the $\mathrm{Ce}$ host ions. The last contribution arises from the RKKY coupling between the $\mathrm{Ce}$ and $\mathrm{Gd}$ ions, which transfers the Ce fluctuations to the Gd site.

The ESR relaxation of an impurity due to (stable) excited configurations of the host ions can be calculated using the Bloch-Redfield-Wangsness formalism, ${ }^{18}$ in analogy with impurity relaxation in Van-Vleck compounds. ${ }^{11}$ Then the relaxation rate can be written as
$\Delta H=\frac{\pi}{2 \hbar^{2}}\left[\partial\left(g_{J}-1\right)\right]^{2}\left[k_{y y}\left(\omega_{0}\right)+k_{x x}\left(\omega_{0}\right)+2 k_{z z}(0)\right]$,

where $\omega_{0}$ is the impurity resonance frequency. $k_{q q}(\omega)(q=x, y, z)$ is the Fourier transform of the spectral functions defined as

$k_{q q}(\omega)=\frac{1}{2 \pi} \int_{-\infty}^{\infty}\left\langle\sum_{k=1}^{z_{0}} \delta J_{q}^{(k)}(t) \sum_{k=1}^{z_{0}} \delta J_{q}^{(k)}\right\rangle e^{i \omega t} d t$.

Here $\left\langle>\right.$ denotes a thermal average and $\delta J_{q}^{(k)}$ the fluctuation in the $q$ component of the total angular momentum of $\mathrm{Ce}$ due to the interconfigurational fluctuations, defined as

$$
\delta J_{q}^{(k)}=J_{q}^{(k)}-\left\langle J_{q}^{(k)}\right\rangle .
$$

In the absence of pair correlations, i.e., terms like $\left\langle\delta J_{q}^{(i)}(t) \delta J_{q}^{(j)}\right\rangle$ with $i \neq j$, Eq. (8) is reduced to the form

$$
k_{q q}=z_{0} K_{q q}(\omega)
$$

with $K_{q q}(\omega)$ defined as

$$
K_{q q}(\omega)=\frac{1}{2 \pi} \int_{-\infty}^{\infty}\left\langle\delta J_{q}(t) \delta J_{q}\right\rangle e^{i \omega t} d t
$$

In the present case, the $\mathrm{Ce}$ interconfigurational fluctuation frequency, $\Delta \omega$, is much greater than the impurity resonance frequency, $\omega_{0}$. Physically for our model it means that for a very short correlation time, the spectral density of the fluctuating field is "white" to the frequencies far above the resonance frequency. So that if we view the problem from a rotating frame (that is one where the magnetization is rotating at the Larmor frequency), the $x, y$, and $z$ directions are equivalent and the effects of the external magnetic field in the fluctuation spectra can be neglected. In this case we have that

$$
K_{x x}\left(\omega_{0}\right) \cong K_{x x}(0) ; K_{y y}\left(\omega_{0}\right) \cong K_{y y}(0)
$$

and for cubic symmetry

$$
K_{x x}(0)=K_{y y}(0)=K_{z z}(0) \text {. }
$$

With these assumptions the calculation of spectral functions (see Ref. 11) leads to the following expression for the linewidth:

$\Delta_{I F}=\frac{2 \pi}{\hbar^{2}}\left[\partial\left(g_{J}-1\right)\right]^{2} z_{0} F(0) \sum_{n \alpha} p_{n}\left|\left\langle n \alpha\left|J_{z}\right| n \alpha\right\rangle\right|^{2}$,

where $|n \alpha\rangle$ and $E_{n}$ are the eigenvectors and eigenvalues of $H, F(0)$ a frequency distribution function due to the finite width of the $\mathrm{Ce} 4 f^{n}$ states, and $p_{n}$ $=\exp \left(-E_{n} / T\right) / Z$, with $Z=\Sigma_{n^{\prime}} \exp \left(-E_{n^{\prime}} / T\right)$.

$\mathrm{Ce}$ ions fluctuate between the $4 f^{0}$ and the $4 f^{1}$ configurations. If we assume the $4 f^{0}$ configuration as the ground state (with $J_{z}=0$ ), the contribution of the excited $4 f^{1}$ configuration to the impurity linewidth can be written as

$$
\Delta_{I F}=A e^{-E_{\mathrm{ex}} / T}
$$

where

$$
A=\left(2 \pi / \hbar^{2}\right)\left[\partial\left(g_{J}-1\right)\right]^{2} z_{0} F(0)\left|\left\langle 1 \alpha\left|J_{z}\right| 1 \alpha\right\rangle\right|^{2}
$$


and $E_{\mathrm{ex}}$ is the excitation energy required to delocalize a single electron from the $\mathrm{Ce} 4 f^{1}$ configuration.

\section{RESULTS AND DISCUSSION}

Using the expression obtained in Eq. (15) we can calculate the total linewidth of the Gd resonance, given by Eq. (7). Figures 1 and 2 plot the calculated linewidth as a function of the temperature for selected cases of $\mathrm{Ce}_{x} \mathrm{La}_{1-x} \mathrm{Os}_{2}$ and $\mathrm{Ce}_{x} \mathrm{Y}_{1-x} \mathrm{Pd}_{3}$ powered samples. The residual linewidth, which is sample dependent, is not considered in the plots (see figure captions). To fit the data we adjust the parameters $b, A$, and $E_{\mathrm{ex}}$, where $b$ represents the "apparent" Korringa parameter. This is done so because we are not considering the exchange narrowing effect of the fine structure of Gd at low $T^{21}$ As stated previously, here we assume an effective spin $S=\frac{1}{2}$ for the magnetic ion. Rigorously, the linewidth calculations have to consider the contribution of the fine structure associated with the spin $S=\frac{7}{2}$ of Gd. However, the exchange narrowing effect collapses the spectra in a single line, which can be treated by an $S=\frac{1}{2}$ effective spin. This collapse, at low $T$, is more evident for big values of $b$ and the resonance is well described by this effective spin. For the cases where $b$ is small and the fine-structure contribution, not considered here, is important we use an apparent Korringa parameter, associated to the $S=\frac{1}{2}$ effective spin. An example of the last case is the $\mathrm{Gd} \mathrm{di-}$ luted in $\mathrm{CePd}_{3}$ resonance. Comparing our theoretical results with the experimental data we can see that in most of the cases we got an excellent fit. The same calculations have been made for $\mathrm{Ce}\left(\mathrm{Pd}_{1-x} \mathrm{Ag}_{x}\right)_{3}$ and $\mathrm{Ce}\left(\mathrm{Pd}_{1-x} \mathrm{Rh}_{x}\right)_{3}$ with similar results. According to our model, at low $T$ the main contribution to the linewidth is originated in the usual Korringa mechanism. At high temperature the population of the excited $\mathrm{Ce} 4 f^{1}$ configuration is in-

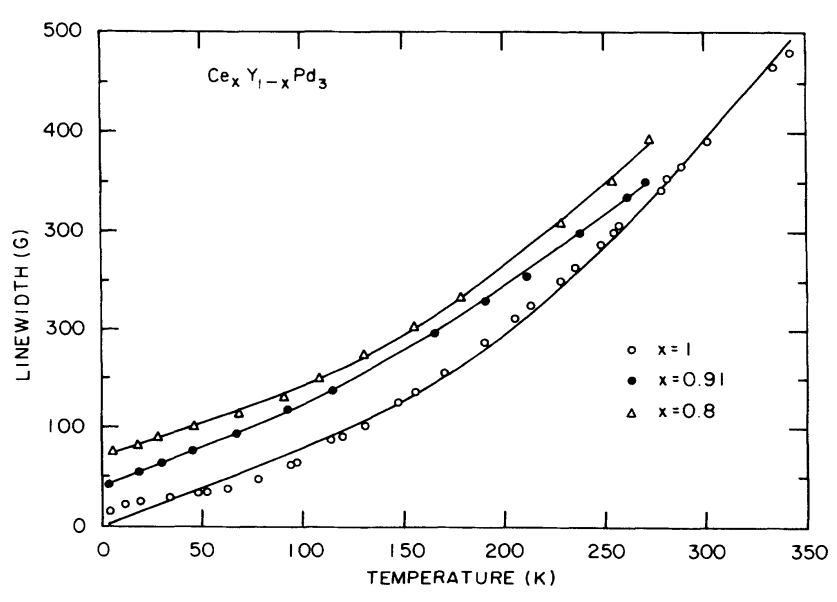

FIG. 1. Temperature dependence of the linewidth for different values of the concentration $x$ in $\mathrm{Ce}_{x} \mathrm{La}_{1-x} \mathrm{Os}_{2}$. The full lines are the plot of the theoretical expression [Eq. (6)]. The fitting parameters can be found in Table $I(a)$. The residual linewidths are not realisitic, for the data was shifted in order to avoid superimposed curves.

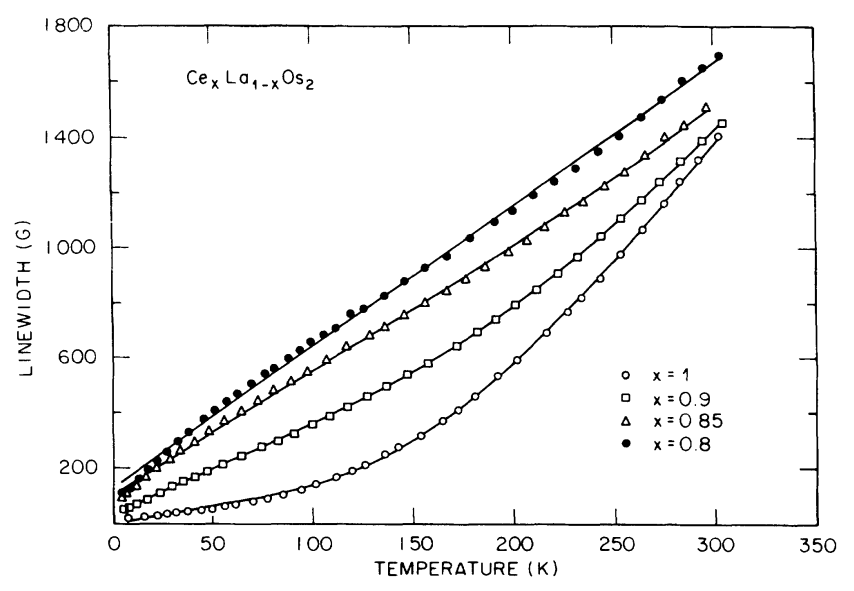

FIG. 2. Same as Fig. 1 for $\mathrm{Ce}_{x} \mathrm{Y}_{1-x} \mathrm{Pd}_{3}$.

creased, and the exponential contribution given by $\Delta_{I F}$ is the most important. The parameters used to fit the experimental data are shown in Table $I$.

Looking at the values of the fitting parameters the effect of the Ce concentration seems clear. The increase in the excitation energy value when the $\mathrm{Ce}$ concentration is reduced agree with the interconfigurational fluctuation model. ${ }^{12-14}$ The model predicts an increase of the excitation energy when we cross from the intermediate valence to the magnetic regime. This is the case, for example, when we go from $\mathrm{CeOs}_{2}$ to $\mathrm{LaOs}_{2}$. On the other hand, the $A$ value depends on the strength of the Ce fluctuation spectra and we can expect an increase with the Ce concentration. The small value of $b$ obtained for high $\mathrm{Ce}$ concentrations, at low $T$, agree with the prediction of the "hybridization hole" model ${ }^{3}$ for Gd diluted in the $\mathrm{CePd}_{3}$ powered sample and with that obtained by $\mathrm{us}^{21}$ for the monocrystalline spectra of the sample compound. This result agrees also with that obtained by Hirst, ${ }^{22}$ for low $T$, but not with the result obtained in Ref. 19, which predicts a higher value for $b$. However, the thermal behavior of the linewidth obtained by the latter authors agree, at least qualitatively, with that obtained here.

TABLE I. Obtained values of the parameters $b, a$, and $E_{\text {ex }}$ that fit Eq. (6) for diluted Gd. The last column shows the experimental value of $E_{\text {ex }}$ published in Ref. 23, to be taken as a comparison.

\begin{tabular}{llccc}
\hline \hline$x$ & $b[G / K]$ & $A[G]$ & $E_{\mathrm{ex}}$ (theor.) & $E_{\mathrm{ex}}$ (expt.) \\
\hline \multicolumn{5}{c}{ (a) $\mathrm{Ce}_{x} \mathrm{La}_{1-x} \mathrm{Os}_{2}$} \\
1 & 1.27 & 9547 & 672 & 500 \\
0.95 & 1.31 & 9580 & 678 & \\
0.9 & 3.28 & 7364 & 849 & \\
0.85 & 4.5 & 3112 & 1132 & \\
0.8 & 5.18 & 94 & 1681 & 360 \\
& \multicolumn{5}{c}{} \\
1 & 0.54 & (b) $\mathrm{Ce}_{x} \mathrm{Y}_{1-x} \mathrm{Pd}_{3}$ & \\
0.91 & 0.69 & 1543 & 576 & \\
0.8 & 0.76 & 642 & 609 & 517 \\
\hline \hline
\end{tabular}


It is important to observe that in contrast to the hybridization hole model, here we have supposed a constant density of states as in a normal metal. The nonlinear contribution to the linewidth, according to the present model, is originated in the exchange interaction of the magnetic impurities with the $\mathrm{Ce}$ ions. Our calculations, regarding the hybridization hole model, permit a quantitative description of the linewidth, but using a lesser number of adjustable parameters. In the other hand, is necessary to point out that for small values of $x$, where the $\Delta_{I F}$ value is small in comparison with the Korringa contribution, the numerical simulation of $\Delta H$ leads to unexpected small values of $E_{\mathrm{ex}}$. However, as we can clearly see in the $\mathrm{Ce}_{x} \mathrm{La}_{1-x} \mathrm{Os}_{2}$ case, $b$ asymptotically approaches the
$\mathrm{LaOs}_{2} b$ value when $x$ goes to 0 .

Note that the values obtained for the excitation energy are, within the experimental error, close to that founded by Sereni, Olcese, and Rizzuto. ${ }^{23}$ This agreement with experimental results supports this interpretation.

\section{ACKNOWLEDGMENTS}

The authors want to acknowledge the International Center of Theoretical Physics, Trieste, Italy, for the hospitality received there, where part of this work was performed. This work was partially supported by CNPQ and FAPESP, Brazil.
${ }^{1}$ J. M. Robinson, Phys. Rep. 51, 1 (1979).

${ }^{2}$ J. Korringa, Physica 16, 601 (1950).

${ }^{3}$ H. Schaeffer and B. Elschner, Z. Phys. 53, 109 (1983).

${ }^{4}$ T. Gambke, B. Elschner, R. Kremer, and M. Schanz, J. Magn. Magn. Mater. 36, 124 (1983).

${ }^{5}$ C. Y. Huang and K. Sugawara, J. Low-Temp. Phys. 28, 229 (1977).

${ }^{6}$ H. Alloul, Physica 86-88B, 449 (1977).

${ }^{7}$ D. E. MacLaughlin, O. Penna, and M. Lysak, Phys. Rev. B 23, 1039 (1981).

${ }^{8}$ A. Narath, in Magnetism, edited by V. H. Suhl (Academic, New York, 1973), p. 150.

${ }^{9}$ B. Giovaninni, P. Pincus, G. Gladstone, and A. J. Heeger, J. Phys. (Paris) Colloq. 321, C1-163 (1971).

${ }^{10}$ M. Mekata, T. Uemura, and T. Kuriyama, Hyperfine Interact. 10, 833 (1981).

${ }^{11}$ D. Davidov, V. Zevin, R. Levin, D. Shaltiel, and K. Baberschke, Phys. Rev. B 15, 2771 (1977).

${ }^{12}$ L. L. Hirst, Phys. Kondens. Mater. 11, 255 (1970).

${ }^{13}$ L. L. Hirst, in Magnetism and Magnetic Materials (San Francisco, 1974), Proceedings of the 20th Annual Conference on Magnetism and Magnetic Materials, AIP Conf. Proc. No. 24, edited by C. D. Graham, G. H. Lander, and J. J. Rhyne (AIP, New York, 1975).

${ }^{14}$ L. L. Hirst, in Valence Instabilities and Narrow Band Phenom- ena, edited by R. D. Parks (Plenum, New York, 1977), p. 3.

${ }^{15} \mathrm{D}$. K. Wohlleben, in Proceedings of the International Conference on Valence Fluctuations in Solids, edited by L. Falicov, W. Hanke, and M. B. Maple (North-Holland, Amsterdam, 1981).

${ }^{16}$ E. R. Bauminger, D. Froindlich, I. Nowik, S. Ofer, I. Felner, and I. Mayer, Phys. Rev. Lett. 30, 1053 (1973).

${ }^{17}$ B. C. Sales and D. Wohlleben, Phys. Rev. Lett. 35, 18 (1975); 35, 1240 (1975).

${ }^{18}$ A. G. Redfield, Adv. Magn. Res. 1, 1 (1965); IBM J. Res. Dev. 1, 19 (1957), see also C. P. Slichter, Principles of Magnetic Resonance, edited by M. Cardona, P. Fulde, and H. J. Queisser, Springer Series in Solid State Sciences, 2nd ed., Vol. 1 (Springer, New York, 1978).

${ }^{19}$ M. M. Ochi and O. L. T. de Menezes, Solid State Commun. 75, 355 (1990).

${ }^{20}$ T. Gambke and T. Elschner, J. Phys. (Paris) Colloq. 40, C5331 (1979).

${ }^{21}$ P. A. Venegas and G. E. Barberis, Solid State Commun. 58, 447 (1987)

${ }^{22}$ T. Gambke, B. Elschner, and L. L. Hirst, Phys. Rev. Lett. 40, 1290 (1978).

${ }^{23}$ J. G. Sereni, G. L. Olcese, and C. Rizzuto, J. Phys. (Paris) Colloq. 40, C5-337 (1979). 